Louisiana State University

LSU Digital Commons

$10-15-2004$

\title{
Membrane lipid biosynthesis in Chlamydomonas reinhardtii: Ethanolaminephosphotransferase is capable of synthesizing both phosphatidylcholine and phosphatidylethanolamine
}

\author{
Wenyu Yang \\ Louisiana State University \\ James V. Moroney \\ Louisiana State University \\ Thomas S. Moore \\ Louisiana State University
}

Follow this and additional works at: https://digitalcommons.Isu.edu/biosci_pubs

\section{Recommended Citation}

Yang, W., Moroney, J., \& Moore, T. (2004). Membrane lipid biosynthesis in Chlamydomonas reinhardtii: Ethanolaminephosphotransferase is capable of synthesizing both phosphatidylcholine and phosphatidylethanolamine. Archives of Biochemistry and Biophysics, 430 (2), 198-209. https://doi.org/ 10.1016/j.abb.2004.07.016

This Conference Proceeding is brought to you for free and open access by the Department of Biological Sciences at LSU Digital Commons. It has been accepted for inclusion in Faculty Publications by an authorized administrator of LSU Digital Commons. For more information, please contact ir@lsu.edu. 


\title{
Membrane lipid biosynthesis in Chlamydomonas reinhardtii: ethanolaminephosphotransferase is capable of synthesizing both phosphatidylcholine and phosphatidylethanolamine ${ }^{\text {证, 弥访 }}$
}

\author{
Wenyu Yang, James V. Moroney, Thomas S. Moore* \\ Department of Biological Sciences, Louisiana State University, Baton Rouge, LA 70803, USA
}

Received 23 April 2004, and in revised form 15 July 2004

Available online 14 August 2004

\begin{abstract}
Phosphatidylethanolamine, but not phosphatidylcholine, is found in Chlamydomonas reinhardtii. A cDNA coding for diacylglycerol: CDP-ethanolamine ethanolaminephosphotransferase (EPT) was cloned from $C$. reinhardtii. The $C$. reinhardtii EPT appears phylogenetically more similar to mammalian aminoalcoholphosphotransferases than to those of yeast and the least close to those of plants. Similar membrane topography was found between the $C$. reinhardtii EPT and the aminoalcoholphosphotransferases from mammals, yeast, and plants. A yeast mutant deficient in both cholinephosphotransferase and ethanolaminephosphotransferase was complemented by the $C$. reinhardtii EPT gene. Enzymatic assays of $C$. reinhardtii EPT from the complemented yeast microsomes demonstrated that the $C$. reinhardtii EPT synthesized both PC and PE in the transformed yeast. The addition of either unlabeled CDP-ethanolamine or CDP-choline to reactions reduced incorporation of radiolabeled CDP-choline and radiolabeled CDP-ethanolamine into phosphatidylcholine and phosphatidylethanolamine. EPT activity from the transformed yeast or $C$. reinhardtii cells was inhibited nearly identically by unlabeled CDP-choline, CDP-ethanolamine, and CMP when $\left[{ }^{14} \mathrm{C}\right] \mathrm{CDP}$-choline was used as the primary substrate, but differentially by unlabeled CDP-choline and CDP-ethanolamine when $\left[{ }^{14} \mathrm{C}\right] \mathrm{CDP}$-ethanolamine was the primary substrate. The $K_{\mathrm{m}}$ value of the enzyme for CDP-choline was smaller than that for CDP-ethanolamine. This provides evidence that $C$. reinhardtii EPT, similar to plant aminoalcoholphosphotransferase, is capable of catalyzing the final step of phosphatidylcholine biosynthesis, as well as that of phosphatidylethanolamine in the Kennedy pathway.
\end{abstract}

(C) 2004 Elsevier Inc. All rights reserved.

Keywords: Ethanolaminephosphotransferase; CDP-ethanolamine pathway; Yeast complementation; Chlamydomonas reinhardtii

Phosphatidylethanolamine (PE) is a major phospholipid component in the membranes of eukaryotic cells. In addition, PE can be acylated to form $N$-acylphosphatidylethanolamine which plays a role in protection of membranes during tissue damage and in membrane sta-

\footnotetext{
This research was supported by the National Science Foundation (Grants MCB-9603626 to T.S.M. and IBN-0212093 to J.V.M.).

is is The nucleotide sequence of $C$. reinhardtii EPT reported in this paper has been deposited in the GenBank database (Accession No. AY375472).

* Corresponding author. Fax: +1 2255782597 .

E-mail address: btmoor@1su.edu (T.S. Moore).
}

bilization during seed germination [1,2]. PE is also a precursor of the $N$-acylethanolamine which works as a neurotransmitter in the brain [3]. A role for PE in cytokinesis has been reported in that the redistribution of $\mathrm{PE}$ on the plasma membrane surface may regulate actin cytoskeleton organization and may mediate a coordinated movement between plasma membrane and actin cytoskeleton to achieve successful cell division [4]. Recent evidence also has shown that PE may regulate lipid homeostasis via the sterol regulatory element-binding protein in Drosophila melanogaster [5]. Biosynthesis of $\mathrm{PE}$ in yeast, mammals, and higher plants can take place by three pathways which may differ in importance 
among organisms. In yeast, PE is produced primarily through the decarboxylation of phosphatidylserine (PS) or by an auxiliary cytidine diphosphate (CDP)-ethanolamine pathway [6]. In mammalian cells, PS decarboxylation appears to be the major pathway of $\mathrm{PE}$ production, along with the CDP-ethanolamine pathway and a base exchange reaction [7,8]. In higher plants, all three mechanisms have been reported, although their contributions may differ significantly among plant species [9]. Overall, the CDP-ethanolamine pathway is believed to play the primary role in PE production.

Three enzymes are responsible for the CDP-ethanolamine pathway. These are ethanolamine kinase (EC 2.7.1.82), which catalyzes the phosphorylation of ethanolamine by ATP to produce phosphoethanolamine; CTP: phosphoethanolamine cytidylyltransferase (ECT) (EC 2.7.7.14), which catalyzes the conversion of phosphoethanolamine into CDP-ethanolamine; and ethanolaminephosphotransferase (EPT) (EC 2.7.8.1), which is localized in the endoplasmic reticulum to catalyze the final step for de novo synthesis of PE via transfer of a phosphoethanolamine moiety from CDP-ethanolamine to diacylglycerol [10]. CMP is the second product of this reaction.

An analogous pathway for phosphatidylcholine (PC) biosynthesis, the CDP-choline pathway, involves three enzymes similar to those for PE synthesis and both of these pathways are termed Kennedy pathways [10]. In the Kennedy pathways, both PE and PC are synthesized from a common precursor, $s n$-1,2-diacylglycerol, in the final step, and the reaction is catalyzed by cholinephosphotransferase and/or ethanolaminephosphotransferase, which utilize either CDP-choline or CDP-ethanolamine as the respective phosphoaminoalcohol donor. Since the final steps in both the CDP-choline and CDP-ethanolamine pathways of PC and PE synthesis are catalyzed by similar enzymes, cholinephosphotransferase (CPT) (EC 2.7.8.2) and EPT, they are collectively referred to as aminoalcoholphosphotransferases (AAPTases). Finally, the two final phospholipid products, PE and PC, may be interrelated by PE conversion to PC through sequential methylation [11], or by ethanolamine exchange for a pre-existing PC head group $[7,12]$.

Although the two enzymes, CPT and EPT, seem to be distinct in their activities in PC and PE synthesis of animals $[13,14]$, it is not clear in higher plants whether either or both of these enzymes could utilize both CDPcholine and CDP-ethanolamine as substrates to synthesize both of the products, PC and PE. A number of studies have indicated such a scenario is possible [15-20]. In addition, a human choline/ethanolaminephosphotransferase was found to be able to synthesize both choline- and ethanolamine-containing phospholipids [21].

Yeast has both a cholinephosphotransferase (CPT1) and an ethanolaminephosphotransferase (EPT1) which are encoded by the genetic loci $C P T 1$ and EPT1, respec- tively. CPT1 has been demonstrated to have much less ethanolaminephosphotransferase activity when compared with EPT1, which in turn was shown to have significant cholinephosphotransferase activity [22-25]. A $56 \%$ amino acid identity was found between the yeast EPT1 and CPT1. Both enzymes are found in the endoplasmic reticulum membranes of yeast.

Chlamydomonas reinhardtii has proven useful for a variety of investigations on photosynthesis, chloroplast biogenesis, flagellar structure and function, light perception, and cell-cell recognition, etc. [26]. Relatively few studies have been conducted on its lipid metabolism. C. reinhardtii has a unique lipid composition when compared to that of higher plants [27]. In particular, PC and PS are not present in its membranes. This makes the organism a very useful system for studying the biosynthesis of PE independent of both PC and PS metabolism, which often are intimately related to synthesis of PE. In this study, we report the sequence of a cDNA which encodes the EPT protein in C. reinhardtii. The deduced amino acid sequence of the gene is homologous to both the yeast EPT1 and CPT1 enzymes, with $45 \%$ similarity to the EPT 1 protein and $42 \%$ to CPT1. Very similar hydropathy profiles and transmembrane domain allocations were found between $C$. reinhardtii EPT protein and the yeast EPT1 and CPT1 polypeptides. The expression of this cDNA in a yeast mutant deficient for both CPT1 and EPT1 complemented the yeast mutant, indicating that the cDNA encodes the EPT in C. reinhardtii. This enzyme was found to respond more effectively to CDP-choline than to CDP-ethanolamine as substrates.

\section{Materials and methods}

\section{Materials}

Restriction endonucleases and other DNA modifying enzymes were from New England BioLabs (Beverly, MA, USA). Primers were made by Integrated DNA Technologies (Coralville, IN, USA). $\left[{ }^{14} \mathrm{C}\right] \mathrm{CDP}$-choline was purchased from Amersham $(54 \mathrm{mCi} / \mathrm{mmoL})$. CMP, CDP-ethanolamine, and CDP-choline were from Sigma-Aldrich. All other chemicals were of reagent grade from miscellaneous sources.

\section{Library preparation}

The cDNA library in plasmids was prepared from a core cDNA library in the form of Lambda Zap II phage, which was purchased from the Chlamydomonas Genomics Project (Duke University, NC, USA). The cDNAs in Lambda Zap II phage were transfected into Escherichia coli by using a ZAP-cDNA Synthesis Kit as described by the manufacturer (Stratagene, Valencia, CA, USA). 
Approximately 60,000 colonies of transformed E. coli were pooled for plasmid preparation, which provides a greater than fivefold representation of the total cDNAs in the library. The pooled plasmids from the colonies were used as templates for PCR-based cloning of EPT cDNA.

\section{cDNA cloning}

The BLAST server (http://www.ncbi.nlm.nih.gov/ BLAST/) was used to compare the $C$. reinhardtii expressed sequence tag (EST) database (http://www.biology. duke.edu/chlamy_genome) with Arabidopsis thaliana and Homo sapiens AAPTase protein sequences (GenBank Accession Nos. AAC61768 and AAD25170) to find highly homologous EST clones. Two ESTs (GenBank Accession Nos. BF863705 and BF863706) from a single clone were obtained in the homology search. One EST corresponded to the 5' UTR and partial N-terminal coding sequence while the other is only part of the $3^{\prime}$ UTR of the cDNA. Primers were designed based on the two EST sequences in order to clone the coding region of cDNA from the library described above. The primers that successfully produced the correct cDNA by PCR included the forward primer 5'-GCA GAC TGG TCC GTC CTA CT-3', 5'-GCG AGA GCT AGA ACT CCA GG-3' and reverse primer 5'-CCG CAT TCG ACA ACA CAC CT-3', 5'-ATC ACC GCA CCA CCA ACA G-3'.

\section{Sequence analysis}

The PCR products spanning the complete EPT cDNA coding region were subjected to agarose gel electrophoresis and extracted from the gel with spin columns from a QIAquick gel extraction kit (Qiagen, Valencia, CA, USA). The purified DNA fragments were sequenced on both strands using Applied Biosystems BigDye terminators (version 2.0, Foster City, CA, USA). The degenerate primers for sequencing the cDNAs included the forward primer 5'-TGA TGC TGT CGC TGG TGC-3', 5'-GTC ACA AGG AGC TGG GCA-3', and reverse primers 5'-TGC CCA GCT CCT TGT GAC-3' and 5'-GCA CCA GCG ACA GCA TCA-3', together with the primers used for PCR.

Nucleotide and deduced amino acid sequences were compared with sequences in the databases at the $\mathrm{Na}$ tional Center for Biotechnology Information (NCBI) by using the BLAST program (http://www.ncbi.nlm. nih.gov/BLAST/). Sequence analysis and alignments were obtained by using the multiple sequence alignment program ClustalW from the European Molecular Biology Laboratory-European Bioinfomatics Institute (EMBL-EBI) server (http://www.ebi.ac.uk/clustalw/). The Prosite database from the EBI server (http://ca. expasy.org/prosite/) was used to find putative consensus motifs for specific domains. The ProtScale program was used to predict the hydrophobicity profiles and secondary structures (http://us.expasy.org/tools/protscale.html) based on the method of Kyte and Doolittle and that of Chou and Fasman [28,29]. The protein subcellular localization, ER retention signals, and membrane topology were predicted by the PSORT program (http:// www.psort.org/) and the SMART program (http://smart.embl-heidelberg.de/) [30]. Genomic sequences and intron positions of the $C$. reinhardtii EPT gene were obtained by blasting the $C$. reinhardtii genomic database using the cDNA sequence (http://genome.jgi-psf. org/chlre1/chlre1.home.html).

\section{Yeast strains and growth}

Yeast strains KT1115 (MAT $\alpha$, leu2-3, leu2-112,

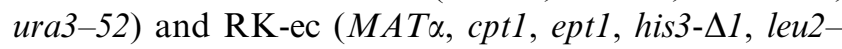
3, leu2-112, and ura2-52) were obtained from Dr. R. Dewey (North Carolina State University, NC). Yeast was cultured in YPD $(1 \%$ yeast extract, $2 \%$ peptone, and $2 \%$ dextrose $[\mathrm{w} / \mathrm{v}])$ or synthetic minimal medium supplemented with proper amino acids at $20 \mathrm{mg} / \mathrm{L} \mathrm{[31].}$ Unless otherwise stated, all yeast operations were carried out according to standard procedures as described [31]. Expression of $C$. reinhardtii EPT cDNA from the $A D H 1$ promoter of yeast expression vector $\mathrm{pDB} 20$ was carried out by culture of the transformed yeast strain in YP-glucose medium ( $1 \%$ yeast extract, $2 \%$ peptone, and $2 \%$ glucose $[\mathrm{w} / \mathrm{v}])$.

\section{Yeast transformation}

Primers with HindIII-adaptor sequences were designed to amplify the complete coding sequence of C. reinhardtii EPT cDNA. After digestion with HindIII, the PCR product was ligated into the HindIII site of a yeast expression vector pDB20 under control of the strong constitutive $A D H 1$ promoter [32]. PCR, restriction endonuclease digestion, and sequencing were used to check for the correct orientation and sequence fidelity of the insert on the vector. This construct, named pDB-EPT, was used to transform the yeast strain RK-ec, which is deficient in both EPT1 and CPT1, by methods previously described [33]. The empty vector pDB20 was also used to transform RK-ec as a negative control.

Screening of yeast strain $R K-$-ec transformed with $C$. reinhardtii EPT cDNA using colony autoradiography

Positive transformants were detected on selective medium (synthetic minimal medium supplemented with histidine and leucine, but without uracil). After two days at $30^{\circ} \mathrm{C}$, transformant colonies began to appear on the 
selective plates. Colonies were then transferred onto $\mathrm{YP}-$ glucose medium for colony autoradiographic assays.

Colony autoradiography was used to screen for yeast colonies complemented in EPT activity as previously described [22]. Confirmation of PC as the product of the enzyme in the colonies tested by autoradiography was achieved by thin layer chromatography (TLC). Briefly, radiolabeled $\mathrm{PC}$ was extracted by soaking the filter discs in $10 \mathrm{~mL}$ chloroform:methanol $(1: 1[\mathrm{v} / \mathrm{v}])$ overnight at $4^{\circ} \mathrm{C}$. The organic phase was recovered by centrifugation at $2000 \mathrm{~g}$ for $10 \mathrm{~min}$ after addition of $5 \mathrm{~mL}$ of $1 \mathrm{~N} \mathrm{HCl}$. After drying, the sample was redissolved in $30 \mu \mathrm{L}$ chloroform and applied to Silica Gel 60 TLC plates (EM Science, Gibbstown, NJ). Development was performed with a solvent of chloroform:methanol:ammonium hydroxide $(29.3 \%[\mathrm{w} / \mathrm{v}]) /$ water $(70: 30: 4: 2[\mathrm{v} / \mathrm{v}])$.

To test if $C$. reinhardtii EPT could synthesize PE in vivo when expressed in yeast strain RK-ec, autoradiographic assays were performed with yeast RK-ec transformed with pDB-EPT and substitution of $\left[{ }^{14} \mathrm{C}\right] \mathrm{CDP}$ ethanolamine as the primary substrate in place of $\left[{ }^{14} \mathrm{C}\right] \mathrm{CDP}$-choline. Confirmation of PE as the product of the enzyme in the colonies tested by autoradiography was achieved by thin layer chromatography.

Ethanolaminephosphotransferase assays with microsomal membranes using $\left[{ }^{14} \mathrm{C}\right] C D P$-choline and $\left[{ }^{14} \mathrm{C}\right] C D P$ ethanolamine as substrates

Chlamydomonas reinhardtii strain cc406 cw15 ( $\left.m t^{-}\right)$ was grown on a rotary shaker into the late exponential phase in $500 \mathrm{~mL}$ Tris-minimal phosphate medium [34] under continuous light at room temperature. Cells were harvested by centrifugation at $1000 \mathrm{~g}$ for $10 \mathrm{~min}$ at $4{ }^{\circ} \mathrm{C}$, washed once with $100 \mathrm{~mL}$ of cold $20 \mathrm{mM}$ Hepes- $\mathrm{KOH}$, $\mathrm{pH} 7.2$, and once with $25 \mathrm{~mL}$ GME buffer $(20 \%$ [v/v] glycerol, $50 \mathrm{mM}$ Mops- $\mathrm{NaOH}, \mathrm{pH} 7.5$, and $1 \mathrm{mM}$ EDTA). The pellet was resuspended in $2 \mathrm{~mL}$ GME buffer, and cells were disrupted by vortexing at high speed with $4 \mathrm{~mL}$ of glass beads $(425-600 \mu \mathrm{m})$ for two 1 -min periods, with the sample being held on ice for $1 \mathrm{~min}$ between vortexing periods. The resulting homogenate was centrifuged at $14,000 \mathrm{~g}$ for $15 \mathrm{~min}$ at $4{ }^{\circ} \mathrm{C}$ to remove the unbroken cells and debris. The supernatant was adjusted to a final volume of $20 \mathrm{~mL}$ with GME buffer and centrifuged at $100,000 \mathrm{~g}$ for $1.5 \mathrm{~h}$ at $4{ }^{\circ} \mathrm{C}$. This pellet was resuspended in a total of $0.5 \mathrm{~mL}$ GME buffer using a $15 \mathrm{~mL}$ Tenbroeck glass homogenizer (Pyrex, Corning, $\mathrm{NY}$ ). Aliquots of the suspension were stored at $-80^{\circ} \mathrm{C}$. All centrifugations were performed at $4{ }^{\circ} \mathrm{C}$, and the samples were kept on ice during all other steps of the procedure. The membrane protein was measured by the Bradford method using BSA as the standard [35].

Yeast colonies were inoculated into $200 \mathrm{~mL}$ YP-glucose, grown to an $A_{600}$ of 1.5, and harvested by centrifu- gation at $1000 \mathrm{~g}$ for $10 \mathrm{~min}$. The harvested cells were washed once with $100 \mathrm{~mL}$ distilled water and once with $25 \mathrm{~mL}$ GME buffer followed by centrifugation each time. The final pellet was resuspended in $1 \mathrm{~mL}$ GME buffer. The resuspended cells were disrupted by vortexing at high speed with $2 \mathrm{~mL}$ of glass beads (425-600 microns) for four 1-min periods, with the sample being held on ice for $1 \mathrm{~min}$ between vortexing periods. The microsomal membrane protein was obtained, aliquoted, and stored as described above for the $C$. reinhardtii microsomes.

The $C$. reinhardtii EPT enzyme assay was conducted with $100 \mu \mathrm{g}$ aliquots of microsomal membrane protein from either yeast or C. reinhardtii microsomal preparations. The microsomal membrane protein was incubated in an assay mixture containing $50 \mathrm{mM}$ Mops- $\mathrm{NaOH}$, $\mathrm{pH} 7.5,20 \mathrm{mM} \mathrm{MgCl}_{2}, 1 \mathrm{mM}$ EDTA, and $25 \mu \mathrm{M}$ $\left[{ }^{14} \mathrm{C}\right] \mathrm{CDP}$-choline $(54 \mathrm{mCi} / \mathrm{mmoL})$ or $25 \mu \mathrm{M}\left[{ }^{14} \mathrm{C}\right] \mathrm{CDP}$ ethanolamine $(50 \mathrm{mCi} / \mathrm{mmoL})$. Linear incorporation of radiolabeled substrate into $\mathrm{PC}$ over a $30 \mathrm{~min}$ period was observed with microsomal membranes prepared from the yeast strains KT1115 and RK-ec transformed with pDB-EPT, and C. reinhardtii strain cc406 cw 15 $\left(m t^{-}\right)$. Microsomal membranes isolated from yeast strain RK-ec and RK-ec transformed with pDB20 produced no detectable quantities of $\left[{ }^{14} \mathrm{C}\right] \mathrm{PC}$ or $\left[{ }^{14} \mathrm{C}\right] \mathrm{PE}$ (data not shown). In substrate competition studies, nonradiolabeled CDP-aminoalcohols or CMP was added to different reaction mixtures in a final reaction volume of $100 \mu \mathrm{L}$. After incubation at $30^{\circ} \mathrm{C}$ for $20 \mathrm{~min}, 1 \mathrm{~mL}$ of $0.1 \mathrm{~N} \mathrm{HCl}$, and $2 \mathrm{~mL}$ of chloroform:methanol $(2: 1 \mathrm{dv} /$ v]) were added to stop the reaction and extract radiolabeled PC and PE. TLC was used to confirm the production of radiolabeled $\mathrm{PC}$ and $\mathrm{PE}$ from the enzyme assays as described earlier. The radiolabeled PC and PE produced from microsomal membrane assays were quantified using liquid scintillation spectrometry. The data shown in Figs. 5-7 are from single representative experiments that were independently repeated three times.

\section{$K_{m}$ determination of EPT for CDP-choline and CDP- ethanolamine}

EPT enzyme assays were conducted as above using the microsomal proteins prepared from either C. reinhardtii strain cc406 cw15 $\left(m t^{-}\right)$or yeast RK-ec transformed with pDB-EPT. One hundred micrograms of microsomal membrane protein was incubated in $100 \mu \mathrm{L}$ assay mixture containing $50 \mathrm{mM}$ Mops- $\mathrm{NaOH}$, $\mathrm{pH} 7.5,20 \mathrm{mM} \mathrm{MgCl}, 1 \mathrm{mM}$ EDTA, and $\left[{ }^{14} \mathrm{C}\right] \mathrm{CDP}-$ choline $(54 \mathrm{mCi} / \mathrm{mmoL})$ or $\left[{ }^{14} \mathrm{C}\right] \mathrm{CDP}$-ethanolamine $(50 \mathrm{mCi} / \mathrm{mmoL})$ at concentrations of $10,30,50,70$, and $100 \mu \mathrm{M}$. The $K_{\mathrm{m}}$ values of the enzyme for CDP-choline and CDP-ethanolamine were obtained from replots of the intercepts on the ordinate against the reciprocal of the concentration of the nonvaried substrate [36]. 


\section{Southern blot analysis}

Genomic DNA from C. reinhardtii wild type strain 137C was isolated as described [37]. Five micrograms of genomic DNA was digested with restriction endonucleases (New England BioLabs), resolved on a 0.8\% (w/ v) agarose gel, transferred to a Nytran membrane (Schleicher and Schuell, Keene, New Hampshire), and hybridized with a radiolabeled probe derived from the coding sequence of the $C$. reinhardtii EPT cDNA by PCR. The DNA transfer, hybridization, and washes were as previously described [38].

\section{Results}

Nucleotide sequence analysis of the EPT gene and its $c D N A$

The cDNA of $C$. reinhardtii EPT gene contains an ORF that encodes a 383 amino acid polypeptide (GenBank accession number for nucleotide sequence is AY375472). C. reinhardtii EPT (cEPT) protein is $45 \%$ similar to the yeast EPT1 polypeptide and $42 \%$ similar to the yeast CPT1 protein at the predicted amino acid level. The identity and similarity of $C$. reinhardtii EPT to the proteins of other organisms are similar to those of yeast (Table 1). Relatively high similarities in AAPTase were found among higher plant species, as well with human and mouse. An alignment of AAPTase protein sequences from $C$. reinhardtii, mammals, and plants was performed (Fig. 1A). A signature sequence, DGKQARRTGTSSPLGQLFDHGCD, was found in the predicted amino acid sequence of cEPT, which aligns well with the consensus sequence associated with CDPalcohol phosphatidyltransferases, D-G-x(2)-A-R-x(8)G-x(3)-D-x(3)-D. Proteins in this family are involved in phospholipid biosynthesis and share the property of catalyzing the displacement of CMP from a CDP-alcohol by a second alcohol, with the formation of a phosphodiester bond and concomitant breaking of a phosphoride anhydride bond [25,39]. These enzymes include diacylglycerol cholinephosphotransferase (EC 2.7.8.2), phosphatidylglycerophosphate synthase (EC 2.7.8.5), PS synthase (EC 2.7.8.8), and phosphatidylinositol synthase (EC 2.7.8.11). A conserved sequence region in cEPT at positions 76-179 was also identified based on sequence homologies with other AAPTases (Fig. 1A). Similar conserved regions among the AAPTases have been proposed to contribute to the formation of the active site of the enzymes in the family of CDP-alcohol phosphatidyltransferases $[25,39]$. This region is predominantly hydrophilic but also contains one or two amphipathic structures which form $\alpha$-helices that interact with the membrane on the cytoplasmic side of the ER. These analyses indicate that cEPT clearly is a member of the CDP-alcohol phosphatidyltransferase family. A phylogram based on the sequence alignment demonstrated that the cEPT is more similar to mammalian CPT/EPT than to yeast EPT1 and CPT1 or plant AAPTases (Fig. 1B).

In addition to the above, similarities in membrane-associated topography also are predicted among yeast EPT1, yeast CPT1, human CPT/EPT protein, mouse CPT/EPT, Arabidopsis AtAAPT1 and AtAAPT2, soybean AAPT1, and Brassica rapa AAPT1 and cEPT based on their hydropathy profiles and prediction of secondary structures. Yeast EPT1 and CPT1, soybean AAPT1, Arabidopsis AAPT1 and AAPT2, and human CPT/EPT have been shown to share similar topological structures with seven membrane-spanning regions [1921,24,25], and C. reinhardtii appears to follow this same profile. The parallel patterns of hydrophobicity and transmembrane domain allocations in the amino acid sequences of cEPT, yeast EPT1, and CPT1 are demonstrated in Fig. 2. The locations of the seven apparent transmembrane domains in cEPT polypeptide are: TM1, 45-67; TM2, 176-198; TM3, 218-240; TM4, 261-280; TM5, 290-309; TM6, 322-341; and TM7, $351-373$. These domains are located in very similar positions within the protein sequences of all three polypeptides. These results indicate a high degree of

Table 1

Identity (\%) and similarity (\% in parentheses) among aminoalcoholphosphotransferases of various organisms

\begin{tabular}{|c|c|c|c|c|c|c|c|c|c|}
\hline & cEPT & yEPT1 & $\mathrm{yCPT} 1$ & hCPT/EPT & $\mathrm{mCPT} / \mathrm{EPT}$ & AtAAPT1 & AtAAPT2 & GmAAPT1 & BrAAPT1 \\
\hline $\mathrm{cEPT}$ & - & 25 & 25 & 28 & 29 & 27 & 29 & 27 & 25 \\
\hline yEPT1 & (45) & - & 56 & 24 & 24 & 31 & 30 & 32 & 30 \\
\hline уСРТ1 & $(42)$ & (72) & - & 25 & 25 & 32 & 30 & 30 & 32 \\
\hline hCPT/EPT & (46) & (42) & (43) & - & 95 & 28 & 27 & 28 & 28 \\
\hline $\mathrm{mCPT} / \mathrm{EPT}$ & (46) & (41) & (43) & (95) & - & 27 & 26 & 27 & 27 \\
\hline AtAAPT1 & (43) & (46) & (48) & (44) & (44) & - & 87 & 80 & 97 \\
\hline AtAAPT2 & (43) & (45) & (47) & (45) & (44) & (92) & - & 77 & 87 \\
\hline GmAAPT1 & (42) & (47) & (47) & (43) & (43) & (89) & (85) & - & 80 \\
\hline BrAAPT1 & (42) & (45) & (48) & (44) & (44) & (98) & (92) & (89) & - \\
\hline
\end{tabular}

cEPT, Chlamydomonas reinhardtii, EPT; yEPT1, yCTP1, Saccharomyces cerevisiae yEPT1 and yCPT1; hCPT/EPT, Homo sapiens CPT/EPT; mCPT/ EPT, Mus musculus CPT/EPT; AtAAPT1, AtAAPT2, Arabidopsis thaliana AAPT1 and AAPT2; GmAAPT1, Glycine max AAPT1; and BrAAPT1, Brassica rapa AAPT1. 


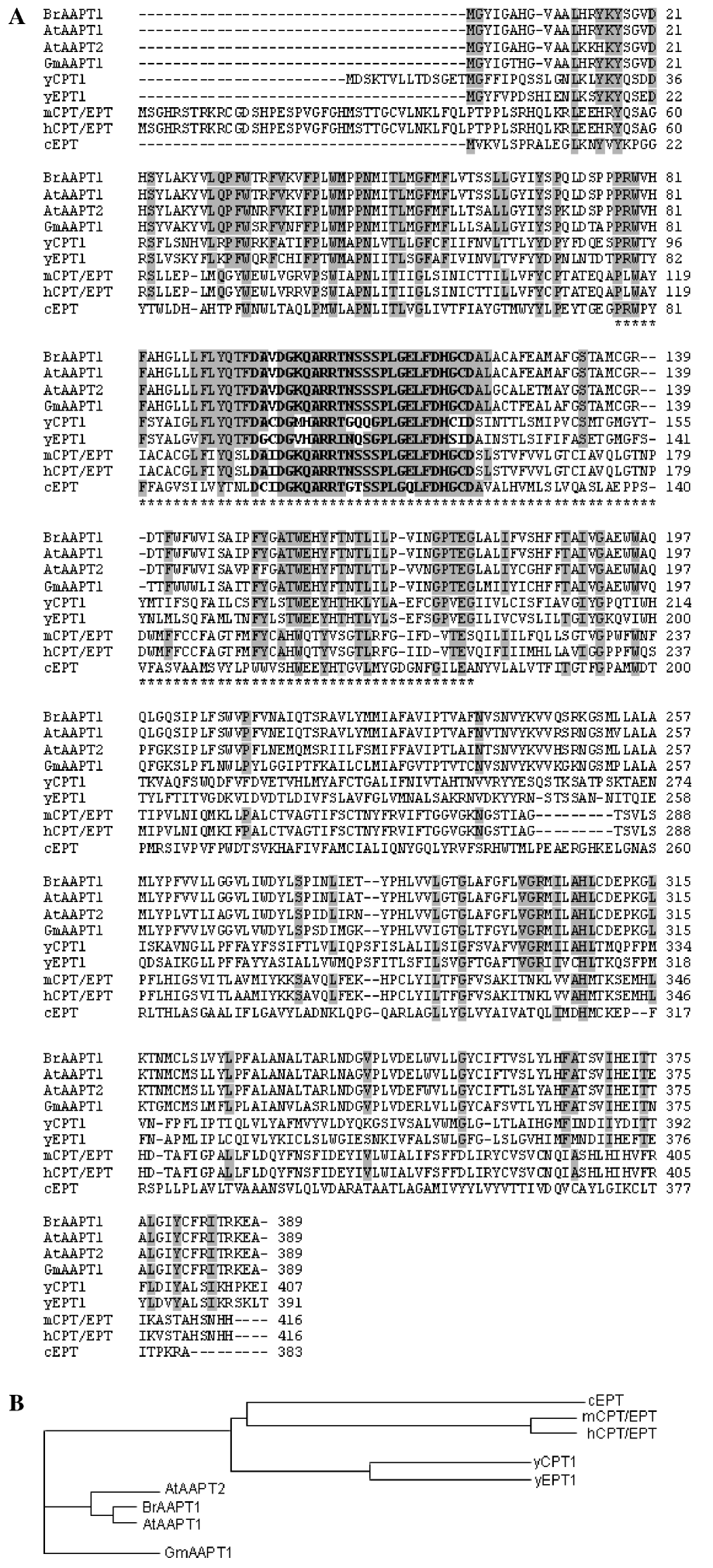

Fig. 1. Sequence alignment of aminoalcoholphosphotransferases in different organisms. (A) Comparison of amino acid sequences among different organisms. cEPT, C. reinhardtii EPT (GenBank Accession No. AAQ83686); yCPT1, yeast CPT1 (AAA63571); yEPT1, yeast EPT1 (AAA63572); AtAAPT1, Arabidopsis AAPT1 (AAC61768); AtAAPT2, Arabidopsis AAPT2 (AAC61769); GmAAPT1, soybean AAPT1 (AAA67719); BrAAPT1, Brassica rapa AAPT1 (AAB53764); hCPT/EPT, human CPT/EPT (AAD25170); and mCPT/EPT, mouse CPT/EPT (AAH21753). The CDP-alcohol phosphatidyltransferase signature in the sequences is in bold. The conserved sequence region shared by all the proteins in the family is indicated with an asterisk. Residue numbers for amino acids are shown on the right. Gaps in alignments are indicated by dashes. Residues that are identical in six or more sequences are shaded in grey. (B) Phylogram of aminoalcoholphosphotransferases in different organisms based on the complete amino acid sequence alignment as shown in (A). 

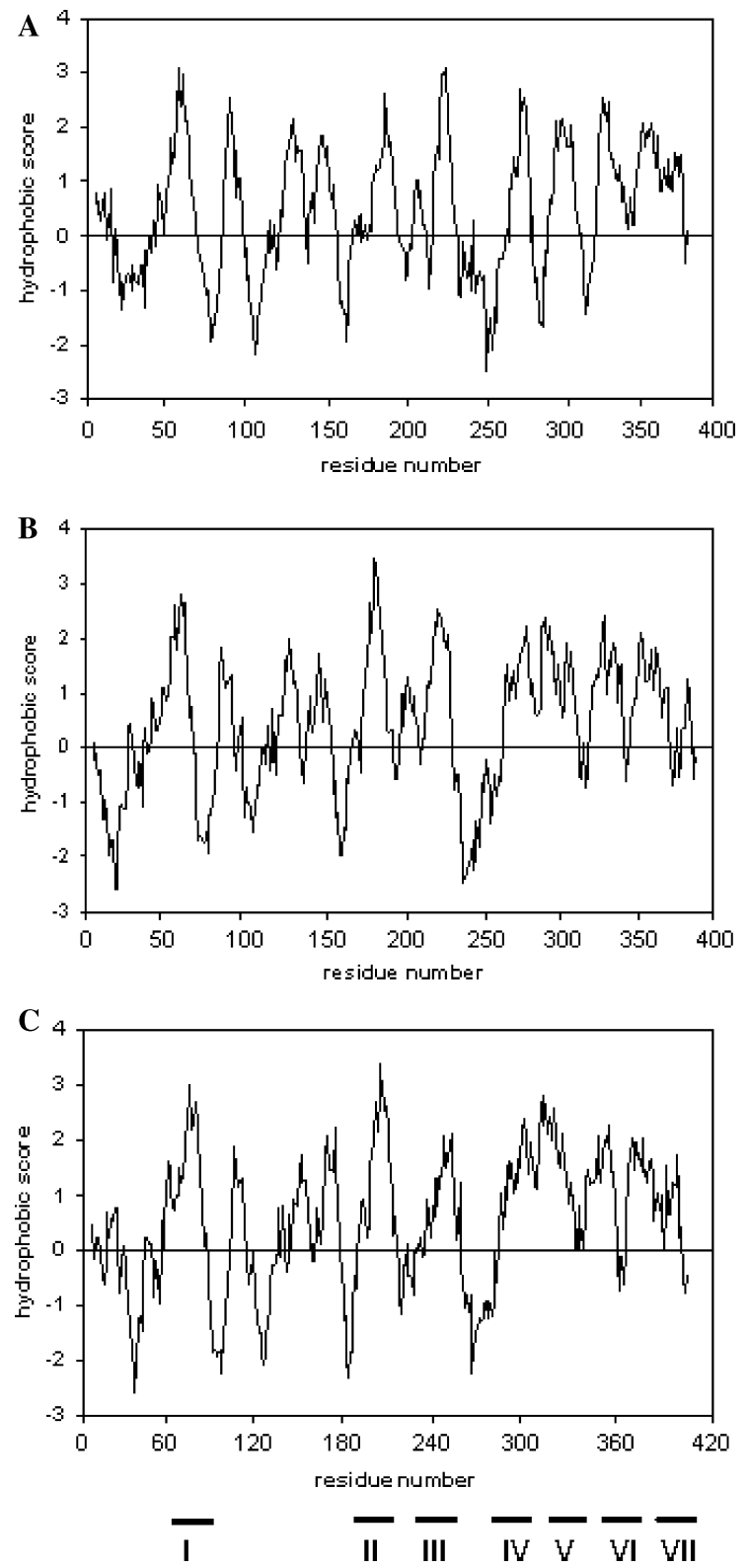

Fig. 2. Hydropathy profile of the predicted C. reinhardtii EPT protein and yeast EPT1 and CPT1. Hydrophobicity was analyzed by the method of Kyte and Doolittle using a window size of nine. Positive values represent hydrophobicity. The locations of transmembrane domains were estimated using PSORT and SMART programs as described in the methods. The seven candidate transmembrane domains for the three polypeptides are indicated as I to VII. (A) C. reinhardtii EPT. (B) Yeast EPT1. (C) Yeast CPT1.

conservation in the membrane-associated topography of the AAPTases in these organisms.

EPT activity has consistently been found associated with the microsomal membrane fraction and most studies show that EPT is located in the ER. Since EPT activity cannot be obtained from biological membranes without the addition of detergents sufficient to completely dissolve the lipid bilayer, it was suggested to be an integral protein $[40,41]$ and this is supported by the
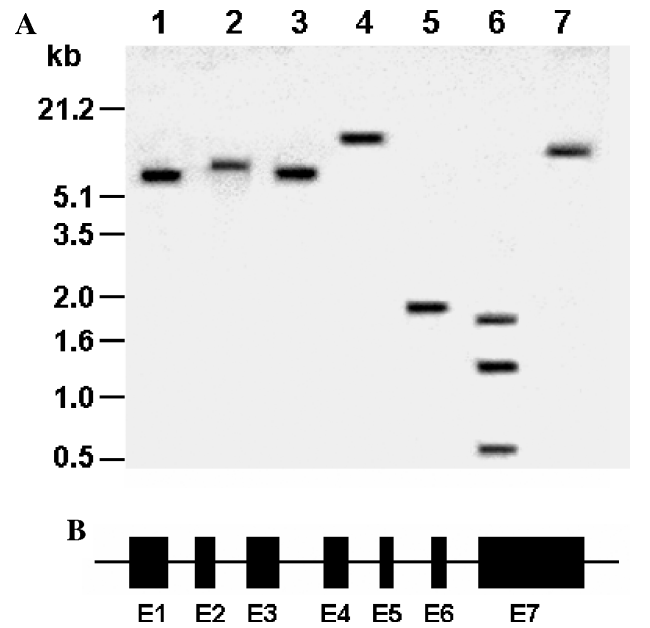

Fig. 3. Southern blot analysis of the EPT gene in the genome of C. reinhardtii wild type strain $137 \mathrm{C}$ and the $E P T$ gene structure. (A) Genomic DNA was digested by EcoRI (lane1), EcoRV (lane 2), HindIII (lane 3), XmnI (lane 4), PvuII (lane 5), PstI (lane 6), and XhoI (lane 7), separated on a $0.8 \%(\mathrm{w} / \mathrm{v})$ agarose gel, transferred to a Nytran membrane, and hybridized to a radiolabeled $1.1 \mathrm{~kb}$ probe from PCR of the coding region of EPT cDNA. (B) Genomic structure of the C. reinhardtii EPT gene. The seven exons are labeled E1-E7. The GenBank Accession No. is AY375473.

topography predictions discussed above. It has been shown that in mammalian cells and yeast, EPT has an absolute requirement for phospholipids, presumably associated with its seven transmembrane domains [21,25,42]. A prediction of $67 \%$ probability of the endoplasmic reticulum as the subcellular localization of the cEPT protein, and $33 \%$ for the plasma membrane, was obtained using the $k$-nearest neighbor $(k-\mathrm{NN})$ algorithm for assessing the probability of localizing at each of the candidate sites [43]. In addition, a KKXX-like motif representative of a type of ER membrane retention signal, TPKR, was found in the C-terminus of the protein (positions 379-382). Moreover, the membrane topology of the EPT protein was predicted to have the N-terminus of the protein located inside the ER membrane. These results all support the cEPT as being an integral membrane protein of the endoplasmic reticulum.

Southern blot analysis of genomic DNA indicated that a single EPT gene is present in the genome of C. reinhardtii, suggesting that there are no other AAPTases in the cells of this species (Fig. 3A). Comparisons of the EPT cDNA sequence with genomic databases provided the complete genomic sequence of the $E P T$ gene from $C$. reinhardtii. The $E P T$ gene of $C$. reinhardtii has six introns and seven exons (Fig. 3B).

Complementation of a yeast strain lacking EPT1 and CPT1 activities

Complementation of a yeast strain deficient in both EPT1 and CPT1 activities was conducted to 
demonstrate the function of the mature cEPT protein encoded by the $C$. reinhardtii EPT gene. Since yeast has both a cholinephosphotransferase (CPT1) and an ethanolaminephosphotransferase (EPT1) and its EPT1 possesses a cholinephosphotransferase activity that is over $50 \%$ as active as its ethanolaminephosphotransferase activity [23], it was essential that the yeast strain used in complementation be mutated at both the EPTI and $C P T 1$ loci. Yeast strains deficient in either EPT1 or CPT1 activity were previously obtained by ethyl methanesulfonate-mediated mutation of yeast cells [22,23]. A double-mutant haploid strain, RK-ec, has been generated from a cross between two single-mutant strains, RH-6D (MAT $\alpha$, cpt1, his3- $\Delta 1$, leu2-3, leu2-112, and ura3-52) for CPT1, and HJ729 (MATa, ept1, and $\mathrm{gal}^{-}$, his3-41, leu2-3, leu2-112, ura3-52, and trp-289) for EPT1 [19]. This double-mutant strain was used for our complementation studies.

The yeast mutant strain RK-ec, deficient in both EPT1 and CPT1 activities, was complemented in colonies from cells transformed with the pDB-EPT construct, while the RK-ec strain without transformation and the RK-ec transformed with empty vector pDB20 had no detectable cholinephosphotransferase or ethanolaminephosphotransferase activity (Fig. 4). Both cholinephosphotransferase and ethanolaminephosphotransferase activities were clearly observed, as a positive control, using a wild type strain of yeast, KT1115. TLC assays of the chloroform-methanol extracted radiolabeled PC and PE from the filter paper demonstrated that more than $95 \%$ of the radiolabeled PC product comigrated with a PC standard (data not shown).
Reduced incorporation of $\left[{ }^{14} C\right] C D P$-choline into $P C$ in the presence of CDP-ethanolamine, CDP-choline, and CMP

To further define this dual substrate utilization, we tested the effects of unlabeled CDP-ethanolamine and CDP-choline on radiolabeled CDP-choline incorporation into PC catalyzed by phosphotransferase contained in microsomal membranes purified from yeast $\mathrm{RK}-\mathrm{ec}$ transformed with pDB-EPT. The results demonstrated that increasing concentrations of either unlabeled CDP-choline or CDP-ethanolamine reduced the synthesis of radiolabeled PC in microsomes from yeast KT1115, RK-ec transformed with pDB-EPT, and C. reinhardtii (Fig. 5). When microsomal membranes from $C$. reinhardtii were similarly tested, the results were comparable to those using microsomal membranes from the yeast strain RK-ec expressing the $C$. reinhardtii EPT gene (Figs. 5B and C). By contrast, in the yeast strain KT1115 a reduction in formation of radiolabeled PC by CDP-ethanolamine was much less than that resulting from addition of CDP-choline (Fig. 5A). As a control, the microsomal membranes from yeast $\mathrm{RK}-\mathrm{ec}$ and RK-ec transformed with pDB20 showed no detectable cholinephosphotransferase activity (data not shown).

When $\left[{ }^{14} \mathrm{C}\right] \mathrm{CDP}$-ethanolamine was used as the primary substrate of the enzyme rather than radiolabeled CDP-choline, similar results were obtained. Increasing concentrations of either unlabeled CDP-choline or CDP-ethanolamine reduced the synthesis of radiolabeled PE by microsomes from yeast RK-ec transformed with pDB-EPT and $C$. reinhardtii cells (Fig. 6). The

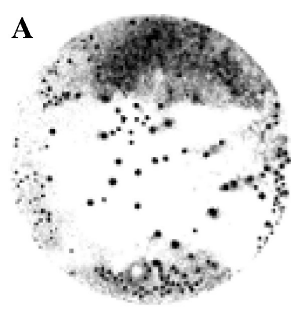

KT 1115

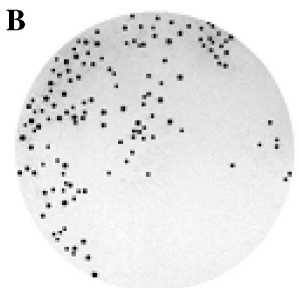

KT1115

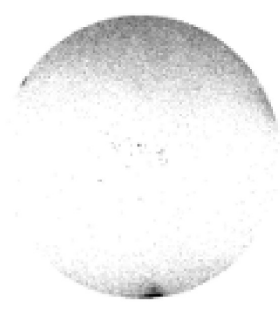

RK-ec

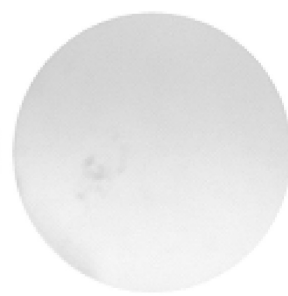

RK-ec

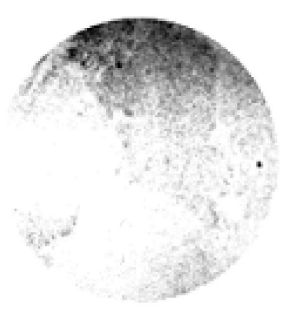

RK-ec: : pDB20

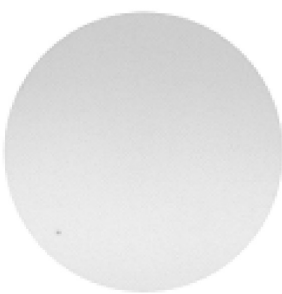

RK-ec:: pDB20

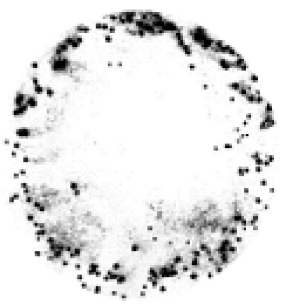

RK-ec: pDB-EPT

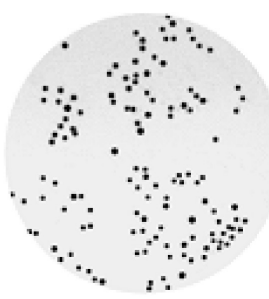

RK-ec: pDB-EPT

Fig. 4. Colony autoradiographic assays for CPT and EPT activity in yeast. Yeast strain KT1115 and RK-ec transformed with pDB20 served as positive and negative controls, respectively. Yeast colonies of all strains used here were grown on $\mathrm{YP}$-glucose medium containing glucose as the sole carbon source. Filters were exposed to X-ray film at $-80{ }^{\circ} \mathrm{C}$ for 4 days before development. (A) $\left[{ }^{14} \mathrm{C}\right] \mathrm{CDP}$-choline was used as substrate in the assay. (B) $\left[{ }^{14} \mathrm{C}\right] \mathrm{CDP}$-ethanolamine was used as substrate in the assay. 


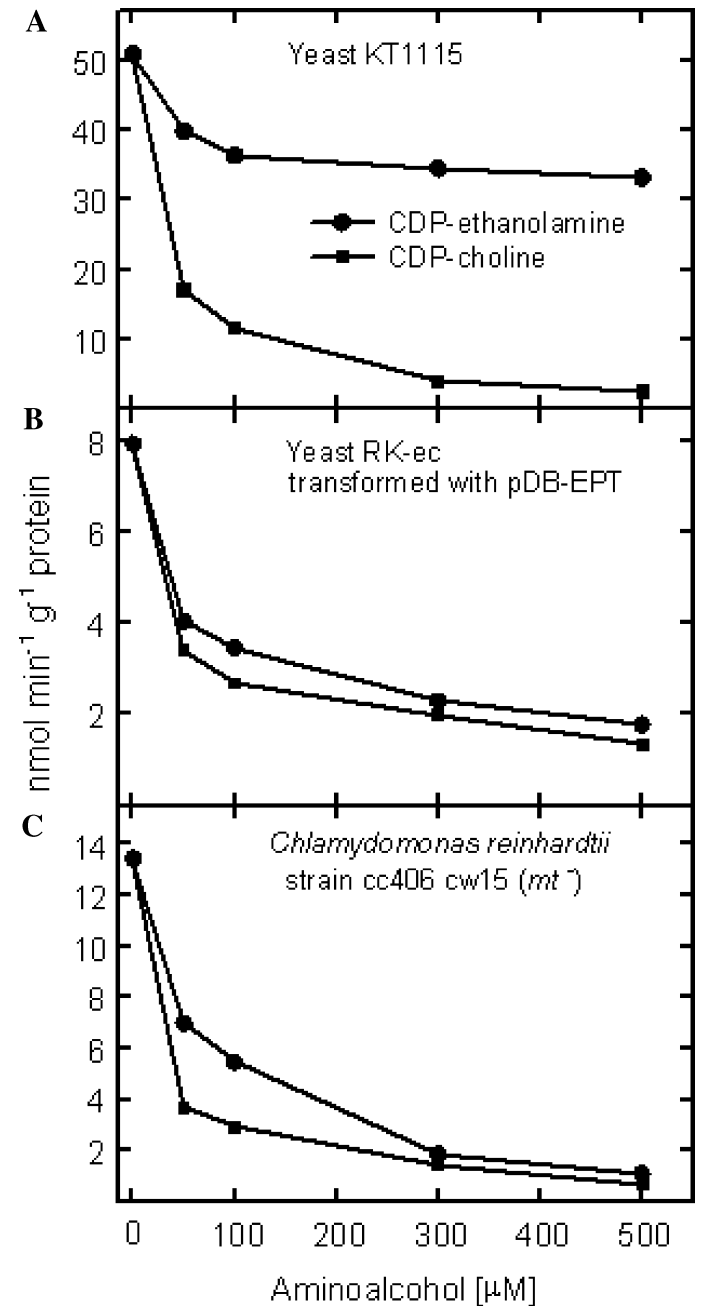

Fig. 5. Effects of CDP-choline and CDP-ethanolamine on the incorporation of $\left[{ }^{14} \mathrm{C}\right] \mathrm{CDP}$-choline into $\mathrm{PC}$ by $C$. reinhardtii $\mathrm{EPT}$ and yeast EPT/CPT enzymes. Microsomal membranes were prepared from yeast strains of KT1115 (wild type for both EPT1 and CPT1 ), RK-ec transformed with pDB-EPT, and C. reinhardtii strain cc406 cw15 $\left(m t^{-}\right)$ as described under "Materials and methods." Enzymatic assays for cholinephosphotransferase were carried out for microsomal membranes at increasing concentrations of unlabeled CDP-ethanolamine

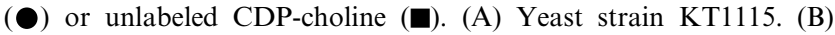
Yeast strain RK-ec transformed with pDB-EPT. (C) C. reinhardtii strain cc406 cw15 $\left(m t^{-}\right)$.

unlabeled CDP-choline appeared more effective in reducing the incorporation of $\left[{ }^{14} \mathrm{C}\right] \mathrm{CDP}$-ethanolamine into PE than CDP-ethanolamine. These data support the hypothesis that the cEPT is more similar to plant phosphotransferases in substrate preferences by being less selective, than it is to the enzyme from other sources, particularly yeast $[17,19,20,25]$.

The effects of increasing concentrations of the watersoluble product of the EPT-catalyzed reaction, CMP, on the cholinephosphotransferase activity of microsomes from yeast KT1115, RK-ec transformed with pDB-EPT, and $C$. reinhardtii strain cc $406 \mathrm{cw} 15\left(m t^{-}\right)$also were tested. Increasing CMP decreased the cholinephosphotrans-
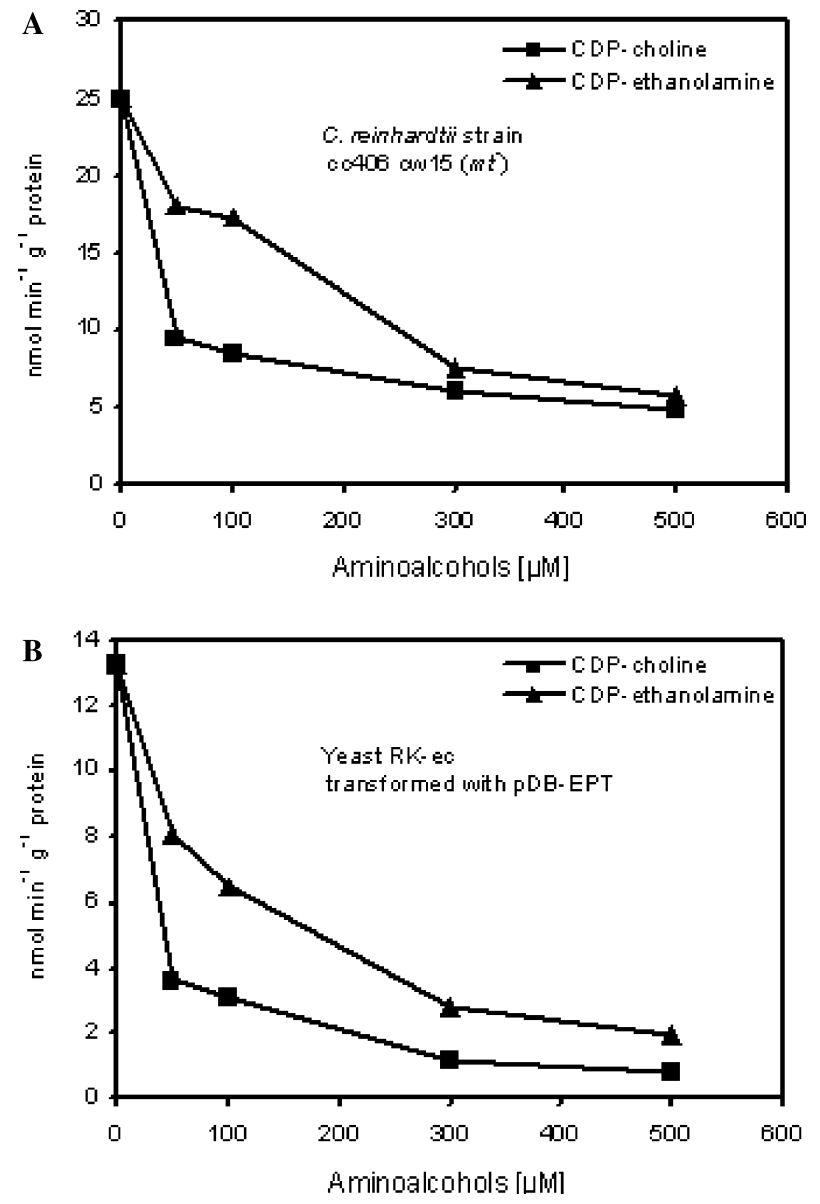

Fig. 6. Effects of CDP-choline and CDP-ethanolamine on the incorporation of $\left[{ }^{14} \mathrm{C}\right] \mathrm{CDP}$-ethanolamine into $\mathrm{PE}$ by C. reinhardtii $\mathrm{EPT}$ and yeast EPT/CPT enzymes. Microsomal membranes were prepared from yeast RK-ec transformed with pDB-EPT and C. reinhardtii strain cc406 cw15 $\left(m t^{-}\right)$as described under "Materials and methods." Enzymatic assays for ethanolaminephosphotransferase were carried out for microsomal membranes at increasing concentrations of unlabeled CDP-ethanolamine ( $\boldsymbol{\Delta})$ or unlabeled CDP-choline (ם). (A) C. reinhardtii strain cc406 cw15 $\left(m t^{-}\right)$. (B) Yeast strain RK-ec transformed with pDB-EPT.

ferase activity of microsomal membranes from yeast RKec transformed with pDB-EPT expressing $C$. reinhardtii $E P T$ gene similarly to the result with microsomal membranes isolated directly from C. reinhardtii cells (Fig. 6). On the other hand, yeast KT1115 microsomes responded much less to increasing concentrations of CMP than did yeast RK-ec transformed with $\mathrm{pDB}-\mathrm{EPT}$ or $C$. reinhardtii EPT. These results show that the algal enzyme is different from that of the yeast, and that the cEPT was not altered in the yeast cells with respect to its substrate preference and sensitivity to CMP.

$K_{m}$ values of $C$. reinhardtii EPT for CDP-choline and CDP-ethanolamine

$K_{\mathrm{m}}$ values of 10.7 and $61 \mu \mathrm{M}$ for CDP-choline and CDP-ethanolamine, respectively, were deduced using 


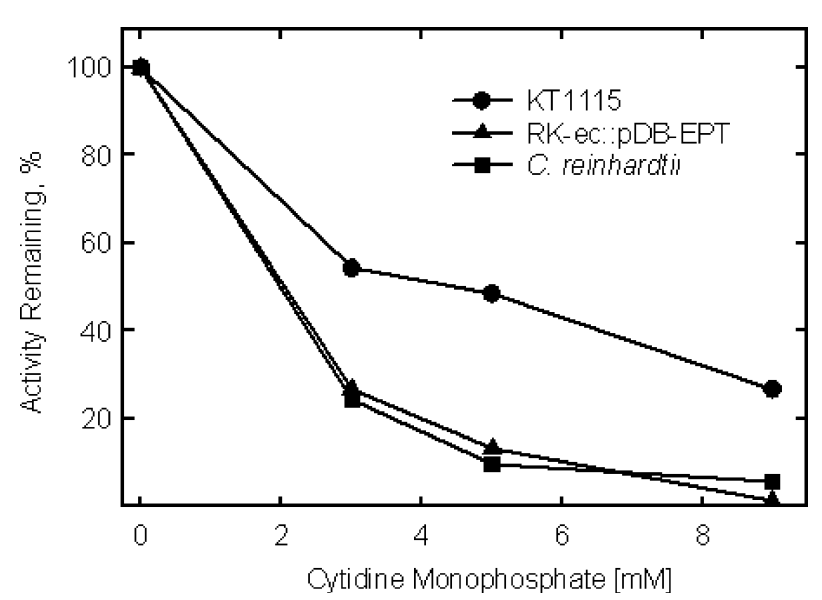

Fig. 7. Effect of CMP on the incorporation of $\left[{ }^{14} \mathrm{C}\right] \mathrm{CDP}$-choline into PC by $C$. reinhardtii EPT and yeast EPT/CPT enzymes. Enzymatic assays for cholinephosphotransferase were conducted in the presence of increasing concentrations of CMP and incubated at $30^{\circ} \mathrm{C}$ for $20 \mathrm{~min}$. Relative activity in percentage is expressed in comparison to a reaction that does not contain CMP. Activities of $100 \%$ were 57.4, 10.4 , and $15.2 \mathrm{nmolmin}^{-1} \mathrm{~g}^{-1}$ protein for microsomal membranes prepared from yeast KT1115 (O), RK-ec transformed with pDB-EPT $(\mathbf{\Delta})$, and $C$. reinhardtii $\mathrm{cc} 406 \mathrm{cw} 15\left(m t^{-}\right)(\boldsymbol{\square})$, respectively.

the microsomal proteins prepared from C. reinhardtii cells as enzyme source. Similarly, $K_{\mathrm{m}}$ values of 5 and $63 \mu \mathrm{M}$ for CDP-choline and CDP-ethanolamine, respectively, were obtained using the microsomal proteins from RK-ec transformed with pDB-EPT. These results indicated that the $C$. reinhardtii EPT has a higher affinity for CDP-choline than for CDP-ethanolamine, and that the enzyme expressed in yeast has characteristics similar to those of C. reinhardtii.

\section{Discussion}

Evidence strongly indicates that plants, animals, and fungi use different types of AAPTases to catalyze the final step of the nucleotide pathways for PE and PC biosynthesis. In animals, it appears that two independent AAPTases primarily use different substrates, CDP-choline and CDP-ethanolamine, for synthesis of PC and $\mathrm{PE}$, respectively $[13,14]$. On the other hand, in higher plants, some evidence indicates that the final step in the Kennedy pathway of $\mathrm{PC}$ and $\mathrm{PE}$ biosynthesis is catalyzed by a single enzyme, while other evidence supports the involvement of separate enzymes in this process [15$18,20,44,45]$.

Indirect evidence has been of particular importance to the above conclusions; especially the observations that a single enzyme can use both CDP-choline and CDP-ethanolamine to synthesize PC and PE, respectively, and that either CDP-choline or CDP-ethanolamine could serve as competitive inhibitors of the other's incorporation in EPT- and CPT-catalyzed reactions. In addition, in higher plants it has been shown that two additional CDP-ethanolamine analogs, CDP-methylethanolamine and CDP-dimethylethanolamine, may also serve as substrates for these same enzymes, leading to production of phosphatidylmethylethanolamine and phosphatidyldimethylethanolamine, which may then be converted to PC by $N$-methyltransferases [46]. The major pathway of PC synthesis seems different among the various species of higher plants that have been studied, including soybean, Lemna paucicostata, carrot, and castor bean endosperm. In soybean, CDP-methylethanolamine appears to be the primary substrate of AAPTase leading to production of PC, while in $L$. paucicostata and castor bean endosperm the major substrate is CDP-choline, and in carrot all three CDPaminoacohols, methylethanolamine, dimethylethanolamine, and choline, are involved in the biosynthesis of PC [46-48].

The current investigation was initiated because C. reinhardtii synthesizes no $\mathrm{PC}$, but does produce $\mathrm{PE}$. This simplifies all interpretations with respect to PE biosynthesis and allows a more direct observation of EPT itself. Indeed, a search of the available $C$. reinhardtii genomic database indicates that no genes coding for the three enzymes in the CDP-choline pathway of PC biosynthesis; choline kinase, CTP: phosphocholine cytidylyltransferase (CCT), and cholinephosphotransferase; occur in the genome of $C$. reinhardtii. Of further interest, no PS decarboxylase gene was found, either. On the other hand, in addition to the enzyme reported here, we also found [49] a CTP: phosphoethanolamine cytidylyltransferase gene in the $C$. reinhardtii genome (AY234844). In light of the data presented in this paper, demonstrating that EPT can recognize both CDP-choline and CDP-ethanolamine, the absence of PC synthesis may primarily be due to the absence of a single enzyme, CCT. In fact, phosphorylcholine appears not to be utilized by microsomal fractions which catalyze the ECT reaction, and unlabeled phosphorylcholine appears not to inhibit the incorporation of radiolabeled phosphorylethanolamine in formation of CDP-ethanolamine by similar fractions (unpublished date). We have not investigated the initial step in these pathways, the kinase reaction, but investigations with this enzyme from yeast and mammalian cells have shown that substrates from either pathway can be utilized by a single enzyme [50]. For these reasons, we conclude that the limiting step for PC synthesis in C. reinhardtii is the absence of CTP: phosphorylcholine cytidylyltransferase (CCT).

In the present study, we found that although the protein sequence of cEPT is more similar to that of mammalian aminoalcoholphosphotransferase than plant AAPTase, it has very high cholinephosphotransferase activity which is typical of plant AAPTase. This high cholinephosphotransferase activity was clearly demonstrated by its use of $\left[{ }^{14} \mathrm{C}\right] \mathrm{CDP}$-choline in the colony autoradiographic assays and the inhibition assays with 
CDP-aminoalcohols and CMP. This result is similar to those obtained when the soybean $A A P T 1$ gene was expressed in the same yeast strain, RK-ec [19].

The determination of the $K_{\mathrm{m}}$ values of the cEPT showed that this enzyme has a higher affinity to CDPcholine than to CDP-ethanolamine, indicating that it may possess higher cholinephosphotransferase activity than ethanolaminephosphotransferase activity. This conclusion was further supported by the greater reduction in corporation of $\left[{ }^{14} \mathrm{C}\right] \mathrm{CDP}$-ethanolamine into $\mathrm{PE}$ by CDP-choline than by CDP-ethanolamine in the substrate competitive assays using microsomal proteins prepared from both RK-ec expressing C. reinhardtii EPT gene and $C$. reinhardtii cells. Since both CDP-choline and CDP-ethanolamine reduced $\left[{ }^{14} \mathrm{C}\right] \mathrm{CDP}$-choline and $\left[{ }^{14} \mathrm{C}\right] \mathrm{CDP}$-ethanolamine incorporation into $\left[{ }^{14} \mathrm{C}\right] \mathrm{PC}$ and $\left[{ }^{14} \mathrm{C}\right] \mathrm{PE}$, respectively, when added to a reaction mixture containing microsomal membranes from transformed strain RK-ec with pDB-EPT or C. reinhardtii, it is clear that the EPT enzymes can utilize either substrate well for synthesis of PC and PE.

By contrast, the yeast KT1115, which possesses both EPT1 and CPT1, demonstrated differential effects of CDP-choline and CDP-ethanolamine on $\left[{ }^{14} \mathrm{C}\right] \mathrm{PC}$ biosynthesis in that inhibition by CDP-ethanolamine was much less than that by CDP-choline. This response is undoubtedly complex, however, due to the presence of the two separate phosphotransferases, as well as differences in their respective total activities and substrate affinities [22,23].

Yeast mutant strains deficient in either EPT1 or CPT1 activity due to single mutations in the EPT1 and $C P T 1$ loci have been characterized in similar competition assays with CDP-choline and CDP-ethanolamine [19]. We have found that the inhibition patterns from KT1115, RK-ec transformed with pDB-EPT, and $C$. reinhardtii differ significantly from those yeast single mutant strains deficient in either EPT1 or CPT1. More specifically, the cholinephosphotransferase activity of yeast deficient in CPT1 and EPT1, but transformed with the C. reinhardtii EPT gene, demonstrates different susceptibilities to CDP-ethanolamine or CDP-choline than do yeast mutants expressing either the CPT1 or $E P T 1$ genes. It was also demonstrated that the cholinephosphotransferase encoded by yeast EPT1 in the mutant strain HJ110, which was deficient in CPT1 activity but contained normal EPT1 activity, had activity reduced to a greater degree by CDP-ethanolamine than by CDP-choline at any concentration when compared with the soybean AAPT1-encoded enzyme [19]. The yeast mutant HJ729, deficient in EPT1 but with normal CPT1 activity, had no reduction in incorporation of CDP-choline as a result of adding nonradioactive CDP-ethanolamine [19]. The inhibition profile of $\left[{ }^{14} \mathrm{C}\right] \mathrm{CDP}$-choline incorporation by the $C$. reinhardtii $E P T$ gene was similar to that observed with soybean $A A P T 1$; that is, the cholinephosphotransferase activity of cEPT was inhibited to a slightly greater extent by CDP-choline than by CDP-ethanolamine in the transformed yeast system.

In summary, the cEPT is similar to mammalian enzymes with respect to protein sequence, but has higher cholinephosphotransferase activity than ethanolaminephosphotransferase activity. It is different from the yeast EPT1 or CPT1. Of the two yeast enzymes, the cEPT appears more closely related to the yeast EPT1, which also has both ethanolaminephosphotransferase and cholinephosphotransferase activities. However, a slight difference between the two enzymes exists, in that the EPT1 of CPT1-deficient yeast demonstrated a higher specificity for CDP-ethanolamine than for CDP-choline. In contrast, cEPT showed a higher specificity for CDP-choline than for CDP-ethanolamine. Since $C$. reinhardtii does not contain PC, this higher native cholinephosphotransferase activity of the $E P T$ gene product may prove interesting for studies on the evolution of $C$. reinhardtii and other algae, particularly with respect to substitution of diacylglyceryltrimethylhomoserine for PC in many species.

\section{Acknowledgment}

We thank Dr. Ralph Dewey at North Carolina State University for providing the yeast strain KT1115, RK-ec, vector pDB20 and for helpful advice in yeast manipulation.

\section{References}

[1] H.H. Schmid, P.C. Schmid, V. Natarajan, Prog. Lipid Res. 29 (1990) 1-43.

[2] K.D. Chapman, Chem. Phys. Lipids 108 (2000) 221-229.

[3] W.A. Devane, L. Hanus, A. Breuer, R.G. Pertwee, L.A. Stevenson, G. Griffin, D. Gibson, A. Mandelbaum, A. Etinger, R. Mechoulam, Science 258 (1992) 1946-1949.

[4] M. Umeda, K. Emoto, Chem. Phys. Lipids 101 (1999) 81-91.

[5] I.Y. Dobrosotskaya, A.C. Seegmiller, M.S. Brown, J.L. Goldstein, R.B. Rawson, Science 296 (2002) 879-883.

[6] M.L. Greenberg, J.M. Lopes, Microbiol. Rev. 60 (1996) 1-20.

[7] T. Miura, J. Kanfer, Arch. Biochem. Biophys. 175 (1976) 654 660.

[8] C. Kent, Annu. Rev. Biochem. 64 (1995) 315-343.

[9] A.J. Kinney, in: T.S. Moore (Ed.), Lipid Metabolism in Plants, CRC Press, Boca Raton, 1993, pp. 259-284.

[10] E.P. Kennedy, S.B. Weiss, J. Biol. Chem. 222 (1956) 193-214.

[11] G. Hubscher, R.R. Dils, W.F. Pover, Biochem. Biophys. Acta 36 (1959) 518-528.

[12] S. Shin, T.S. Moore, Plant Physiol. 93 (1990) 148-153.

[13] R.M. Bell, R.A. Coleman, Annu. Rev. Biochem. 49 (1980) 459 487.

[14] A.K. Percy, M.A. Carson, J.F. Moore, C.J. Waechter, Arch. Biochem. Biophys. 230 (1984) 69-81.

[15] B.A. Macher, J.B. Mudd, Plant Physiol. 53 (1974) 171-175.

[16] J.M. Lord, Biochem. J. 151 (1975) 451-453.

[17] S.A. Sparace, L.K. Wagner, T.S. Moore, Plant Physiol. 67 (1981) 922-925. 
[18] A.M. Justin, C. Demandre, A. Tremoliers, P. Mazliak, Biochim. Biophys. Acta 836 (1985) 1-7.

[19] R.E. Dewey, R.F. Wilson, W.P. Novitzky, J.H. Goode, Plant Cell 6 (1994) 1495-1507.

[20] J.H. Goode, R.E. Dewey, Plant Physiol. Biochem. 37 (1999) 445457.

[21] A.L. Henneberry, C.R. McMaster, Biochem. J. 339 (1999) 291298.

[22] R.H. Hjelmstad, R.M. Bell, J. Biol. Chem. 262 (1987) 3909-3917.

[23] R.H. Hjelmstad, R.M. Bell, J. Biol. Chem. 263 (1988) 1974819757.

[24] R.H. Hjelmstad, R.M. Bell, J. Biol. Chem. 265 (1990) 1755-1764.

[25] R.H. Hjelmstad, R.M. Bell, J. Biol. Chem. 266 (1991) 5094-5103.

[26] H.H. Harris, Annu. Rev. Plant Physiol. Plant Mol. Biol. 52 (2001) 363-406.

[27] C. Giroud, A. Gerber, W. Eichenberger, Plant Cell Physiol. 29 (1988) 587-595.

[28] J. Kyte, R.F. Doolittle, J. Mol. Biol. 157 (1982) 105-132.

[29] P.Y. Chou, G.D. Fasman, Adv. Enzymol. 47 (1978) 45-148.

[30] I. Letunic, R.R. Copley, S. Schmidt, F.D. Ciccarelli, T. Doerks, J. Schultz, C.P. Ponting, P. Bork, Nucleic Acids Res. 32 (2004) D142-D144.

[31] C. Guthrie, G.R. Fink, Guide to Yeast Genetics and Molecular Biology: Methods in Enzymology, vol. 194, Academic Press, New York, pp.3-21 1991.

[32] D.M. Becker, J.D. Fikes, L. Guarente, Proc. Natl. Acad. Sci. USA 88 (1991) 1968-1972.

[33] D. Gietz, A.St. Jean, R.A. Woods, R.H. Schiestl, Nucleic Acids Res. 20 (1992) 1425.
[34] H.H. Harris, The Chlamydomonas Sourcebook: A Comprehensive Guide to Biology and Laboratory Use, Academic Press, San Diego California, 1989 pp. 25-40.

[35] M.M. Bradford, Anal. Biochem. 72 (1976) 248-254.

[36] J.R. Florini, C.S. Vestling, Biochim. Biophys. Acta 25 (1957) 575 578.

[37] J.D. Rochaix, J. Mol. Biol. 126 (1978) 597-617.

[38] J. Sambrook, E.F. Fritsch, T. Maniatis, Molecular Cloning: A Laboratory Manual, Cold Spring Harbor Laboratory Press, Cold Spring Harbor, New York, 1989 pp. 9.47-9.58.

[39] J. Nikawa, T. Kodaki, S. Yamashita, J. Biol. Chem. 262 (1987) 4876-4881.

[40] J.E. Vance, D.E. Vance, J. Biol. Chem. 263 (1992) 5898-5909.

[41] A. Mancini, R. Roberti, L. Binaglia, A.E. Missiri, L. Freysz, Membrane Biochem. 10 (1993) 43-52.

[42] C.R. McMaster, S.C. Morash, R.M. Bell, Biochem. J. 313 (1996) 729-735.

[43] P. Horton, K. Nakai, Proc. Int. Conf. Intell. Syst. Mol. Biol. 5 (1997) 147-152.

[44] C.W. Dykes, J. Kay, J.L. Harwood, Biochem. J. 158 (1976) 575581.

[45] J.L. Harwood, Phytochemistry 15 (1976) 1459-1463.

[46] M.P. Prud'homme, T.S. Moore, Plant Physiol. 100 (1992) $1527-$ 1535.

[47] A.H. Datko, S.H. Mudd, Plant Physiol. 88 (1988) 854-861.

[48] A.H. Datko, S.H. Mudd, Plant Physiol. 88 (1988) 1338-1348.

[49] W. Yang, C.B. Mason, S.V. Pollock, T. Lavezzi, J.V. Mooney, T.S. Moore, Biochem. J. 382 (2004) 51-57.

[50] K. Ishidate, Biochim. Biophys. Acta 1348 (1997) 70-78. 